\title{
The $q=1$ peak in the mass-ratios for Hipparcos visual binaries
}

\author{
S. Söderhjelm
}

(retired from) Lund Observatory, Box 43, 22100 Lund, Sweden

e-mail: staffan@astrokonsult.se

Received 12 July 2006 / Accepted 23 October 2006

ABSTRACT

\begin{abstract}
Aims. The magnitude differences for Hipparcos visual binaries give important information about the upper part of their mass-ratio distribution, and in previous studies, the author found a narrow peak at $q=1$ This excess of equal-mass, wide pairs has not been confirmed in other studies, and the present work aims to strengthen the Hipparcos results.

Methods. We construct a Galaxy model with binaries of known properties, filter it through a "Hipparcos-like" selection model, and then compare the model with the actual Hipparcos observations. By changing the input distributions in the model, the fit to the observations can be improved, enabling some conclusions about the mass-ratio distribution $f_{q}(q)$ and the distribution of semi-major axes $f_{a}(a)$. The most important weak point is the modelling of the selection effects in the Hipparcos Input Catalog, but by testing different biases, typical effects can be quantified.

Results. From fittings in different color(mass)-intervals, I find that the shapes of the distributions of orbit-sizes and mass-ratios vary with mass, even in the small (1.9-5.5 Msun) mass-sum interval well covered by the Hipparcos data. In particular, the excess of "twins" in $f_{q}(q)$ is definitely present, but the peak amplitude diminishes at higher masses. There is normally also a decrease in the number of binaries with increasing mass-ratio in the interval $0.5<q<0.9$, but below 2 Msun, this slope suddenly reverses, giving a broad peak towards 1.0 below the narrow one. For typical wide orbits $(100-1000 \mathrm{au})$, the $f_{a}(a)$-distribution shows a steeper decline for lower masses, while almost reaching the scale-free $1 / a$ power-law for the highest ones.
\end{abstract}

Key words. binaries: general - binaries: visual - methods: miscellaneous - stars: evolution - stars: formation

\section{Introduction}

The Hipparcos satellite observed reasonably accurate magnitude differences for several thousand visual binaries, and in most cases, these luminosity-ratios are a good indication of the massratios. In two previous reports (Söderhjelm 1997, 2000), I derived a mass-ratio distribution with a sharp peak near $q=1$. Other authors have found such a peak only at much shorter periods (Tokovinin 2000; Halbwachs et al. 2003; Lucy 2006), and it is important to look again at the Hipparcos data (ESA 1997, hereafter HIP). In the first study above, I tried to use complete samples of $\mathrm{A}$ and $\mathrm{F}$ stars, which are then rather small. In the second study, as well as in the present one, I instead compare a model Galaxy with all the Hipparcos observations, giving larger samples, but needing a filtering of the simulated Galaxy data through a model of the instrument and observation process.

An early use of the same basic kind of methodology was the study by Eggleton et al. (1989), using different synthetic models to reproduce the (almost complete) observations of the apparently brightest visual binaries in the sky. Recently, Kouwenhoven et al. (2007), see Kouwenhoven (2006), have made a thorough theoretical clarification of what they call the "S.O.S."(=Simulating Observations of Simulations) method, stressing the importance of recognizing (and simulating) several different kinds of observational biases. In the present study, their "sample bias" is a major problem, since it is very difficult to model the complicated selections into the Hipparcos Input Catalog, governing which stars were included in HIP. The results derived depend on the selection model, but by varying the model, one may try to see what (if any) kind of selections are needed to produce a spurious peak.
Any model Galaxy has to be historic, in the sense that it describes the present stellar content as the result of a star formation history with many successive generations of stars. Binary stellar evolution and galactic dynamics have to be included, and a fully realistic model is bound to become very complex. However, using a number of simplified Galaxy models, it is possible to see how variations in the input mass-ratio and orbit-size distributions of the binaries translate into observable changes in the distribution of separations and magnitude-differences for Hipparcos visual binaries, and consequently to determine at least part of these distributions.

In Sect. 2, I describe the Galaxy model, and in Sect. 3 the selection modelling needed to obtain reasonable all-star HIP model counts. In Sect. 4, I describe the methods used to derive the distributions of semi-major axes and mass-ratios, and then provide the results in Sect. 5. The possible selection biases are discussed in Sect. 6, and in Sects. 7 and 8 I compare the results with earlier studies and theories. Two short appendices list supplementary details.

\section{Modelling the Galaxy}

\subsection{Program basics}

The Galaxy model is a (thick and thin) disk, with plausible scale-lengths and (age-dependent) scale-heights, and with a crude (10 deg resolution) extinction model. The input metallicity distribution is wide and almost age-independent, to give the observed distribution given e.g. in Nordström et al. (2004). Single and binary stars are created over 12 Gyr with a mostly constant rate, and their evolution is followed to give the 
present-epoch observed sky. This model has been used extensively in the Gaia context (Söderhjelm 2005), and a crucial ingredient there was the rapid binary star evolution (BSE) code by Hurley et al. (2002), enabling predictions also about close binaries. In the present study, the binaries studied are long-period and non-interacting, and in this case BSE uses the single-star evolutionary tracks given in Hurley et al. (2000), at (marginally) increased speed. Close binaries are still modelled, however, although they are only observed as unresolved singles.

To create binaries and single stars in a consistent way, the model uses a time-independent IMF close to that given in Kroupa (2001), extending from 0.03 Msun to about 50 Msun. Each mass-lump is given an (ad hoc) $25 \%$ chance of becoming a true single star, but the $75 \%$ majority are split into doubles with some assumed distribution $f(a, q, e)$ of semi-major axis, massratio, and orbital eccentricity. Even if it is clear that this function is in general not separable, one may make the simplistic first assumption $f(a, q, e)=f_{a}(a) \times f_{q}(q) \times f_{e}(e)$. It will be clear from the results below that there are also large mass-dependencies in $f_{a}(a)$ and $f_{q}(q)$, as modelled in some of the runs.

In all the Galaxy model runs, the input distribution of the semi-major axes of the binaries is the full log-normal one of Duquennoy \& Mayor (1991)

$f_{a}(\lg a) \sim N^{\prime}\left(\lg a-\lg a_{m}, \sigma_{a}\right) \quad-3<\lg a[\mathrm{au}]<5$

with $\lg a_{m}$ and $\sigma_{a}$ both close to around 1.5. Here and in the following, $N^{\prime}$ stands for the unnormalized normal distribution $N^{\prime}(m, s) \equiv \exp \left(-0.5(\mathrm{~m} / \mathrm{s})^{2}\right)$.

The input $q$-distribution (with $q<1$ for the ratio of the originally smaller to the larger mass) is again defined for the whole interval $q=0.01-1$, even if only the $q>0.5$ part is compared with observations. Three different parametrizations of $f_{q}(q)$ were used, listed here for completeness. The first version was a wide Gaussian with a narrow $q=1$ peak, viz.

$f_{q}(q) \sim A_{0}+A_{q} N^{\prime}\left(q-q_{m}, \sigma_{q}\right)+A_{p} P(q)$

with (mostly) $P(q) \equiv N^{\prime}(q-1,0.04)$. Later, I used

$f_{q}(q) \sim \begin{cases}N^{\prime}(q-0.5,0.4) & q<0.5 \\ 1-B_{q}(q-0.5)+B_{p} P(q) & q>0.5\end{cases}$

where the low- $q$ part is ad hoc (but not unrealistic), and where the slope $B_{q}$ and the peak amplitude $B_{p}$ could easily be estimated. In the last runs, I used the power-law + peak model as used in the analysis (cf. Sect. 4.2) also as model input for $q>0.5$, viz.

$f_{q}(q) \sim \begin{cases}N^{\prime}(q-0.5,0.4) & q<0.5 \\ (q / 0.5)^{C_{q}}\left(1+C_{p} P(q)\right) & q>0.5\end{cases}$

Although $A_{p}, B_{p}$ and $C_{p}$ all stand for the amplitude of a $q=1$ peak, they differ by the different normalizations in Eqs. (2)-(4). The $C_{p}$ formulation gives the amplitude in relation to the powerlaw background at $q=1$.

The eccentricities are distributed thermally $(\sim e)$ for the largest orbits, turning smoothly into a uniform distribution for $a<10$ au. The tidal evolution built into the BSE-code usually produces a rapid circularization for periods below about 10 days.

In order to make comparisons with the Hipparcos and Tycho observations, there is the standard problem of going from theoretical $\left(M_{\mathrm{bol}}, T_{\mathrm{eff}}\right)$ to observable $\left(M_{V}, V-I, B_{T}-V_{T}\right)$ coordinates, and in view of the many other uncertainties, only rather crude transformations have been used. They are based mainly on data in Flower (1996), with misprint corrections and extensions by P. Nurmi (private comm.) and the author, and on Vol. 1 of HIP.

\subsection{Actual runs}

Because the BSE is used in a simplistic mode (one call for each required age, discarding all intermediate results), the above model is rather slow, with typically several weeks of (PC) computing for an all-sky run. Tables A.1 and A.2 in Appendix A give a list of runs assembled over a ten-month period, with different input parameters for $f_{a}$ and $f_{q}$. The background Galaxy was not identical in all the runs, but none of the changes (in the extinction modelling and the time-evolution of the scale-heights) produced any significantly better fit to the Tycho data (see Sect. 3), and no details are provided.

\section{General star counts}

The Hipparcos satellite observed only the stars in the pre-defined Input Catalog. Most bright stars $(V<7.5)$ are included, but then the completeness becomes successively lower towards a faint limit around $V=12$. An almost complete sample to $V=11$ was simultaneously obtained by the Tycho (TYC) experiment using Hipparcos Star Mapper data (ESA 1997; improved Tycho-2 version in Høg et al. 2000). The Hipparcos Input Catalog is the final result of a complicated merging of some 200+ requests for observations (cf. Vol. 3 of HIP), and the final completeness of Hipparcos, $C_{\mathrm{H}}(b, H p, V-I, \ldots)$, defined as the number of Hipparcos stars divided by all stars of this magnitude and color (e.g. from TYC) can only be obtained empirically (cf. Mignard 2000).

In the present study, I developed two analytical models for $C_{\mathrm{H}}$. One started from the one given in Söderhjelm (2000), using a complex color-equation but a constant slope with respect to galactic latitude. The other one introduced from the outset the magnitude-varying latitude slopes found by Mignard (2000). Both versions have various ad hoc additions, but still mostly use smooth mathematical formulae for something which is not expected to be that simply characterized. Adding to the difficulties are the low numbers of faint Hipparcos stars, as well as the uncertain transformations between the 2 -color $\left(V_{T}, B_{T}\right)$ magnitudes in TYC and the broadband HIP ( $H p)$ magnitudes. Neither of the selection models is able to reproduce the observed completeness counts in detail, but they largely agree, and a final average version was deemed sufficient for our purposes. (There may be extra selection effects related explicitly to the double stars as discussed at length in Sect. 6.)

With the basic assumption that the details of the Galaxy model are not important, the idea is to take a Galaxy modelling (from Tables A.1 and A.2), filter it through the selection model, and then compare the model binary star statistics with the actual Hipparcos observations. Ideally, the Galaxy model should give the observed Tycho star-counts in (reasonably sized) bins of apparent magnitude, color and galactic latitude), but in practice, this works reasonably well ( $\sim 0.1$ dex errors) only for middle $(V-I \approx 0.2-0.7)$ colors, with much larger discrepancies at the blue end. The influence of this poor Galaxy modelling should not be too serious as long as the selection filter gives a correct ratio of Hipparcos to Tycho star numbers. Because $H p$ and $V_{T}$ star counts are not directly comparable, I compare instead the model/obs ratios (since the model gives counts for either magnitude). To decrease some of the random errors, the analysis is made in the nine interlaced color-intervals given in Table 1.

Figure 1 show sample model/observed star-numbers for TYC (showing the "absolute" error) and for HIP (with the selection filter), for the nine color-intervals, as a function of 
Table 1. The $V-I$ limits for the nine interlaced main-sequence color bins used for the analysis. The corresponding TYC $B_{T}-V_{T}$ colors are interpolated from Table 1.3.7 in Vol. 1 of HIP. Also given are the mean masses of the primaries and the systems, as derived from the model runs.

\begin{tabular}{ccccc}
\hline \hline color-bin & $V-I$ & $B_{T}-V_{T}$ & Mpr/Msun & Mtot/Msun \\
\hline C1 & $<0.1$ & $<0.12$ & 3.40 & 5.54 \\
C2 & $0.0-0.2$ & $-0.03-0.21$ & 2.88 & 4.86 \\
C3 & $0.1-0.3$ & $0.12-0.29$ & 2.32 & 3.99 \\
C4 & $0.2-0.4$ & $0.21-0.36$ & 1.93 & 3.38 \\
C5 & $0.3-0.5$ & $0.29-0.45$ & 1.67 & 2.94 \\
C6 & $0.4-0.6$ & $0.36-0.56$ & 1.43 & 2.56 \\
C7 & $0.5-0.7$ & $0.45-0.70$ & 1.26 & 2.28 \\
C8 & $0.6-0.8$ & $0.56-0.84$ & 1.14 & 2.05 \\
C9 & $0.7-0.9$ & $0.70-1.01$ & 1.03 & 1.86 \\
\hline
\end{tabular}

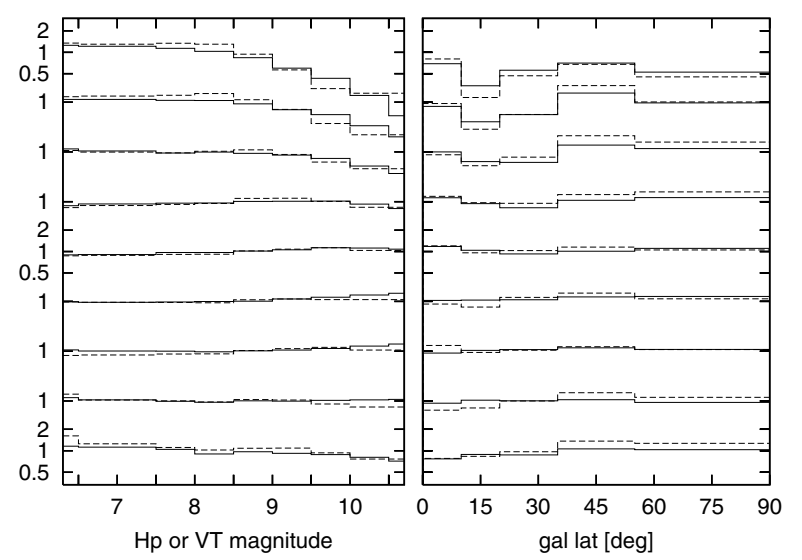

Fig. 1. Comparison of observed and calculated star-counts for a typical (B218) Galaxy model run. The full lines are the TYC model/obs values and the dashed ones the HIP model/obs values, in the nine color-bins of Table 1, with $\mathrm{C} 1$ at the top and $\mathrm{C} 9$ at the bottom. To the left, the data are given as a function of magnitude (using $V_{T}$ for TYC and $H p$ for HIP), and to the right as a function of galactic latitude. The logarithmic scale is indicated for $\mathrm{C} 1, \mathrm{C} 5$ and $\mathrm{C} 9$.

magnitude and of galactic latitude. The deviations at the blue end are large, but the selection model makes the HIP curves follow the TYC ones, meaning that we can make useable predicted HIP-counts from any run of the Galaxy model.

\section{Principles for the double-star analysis}

With the model HIP star-counts normalized to the TYC data, we can concentrate on the main task, the binary star statistics. We want to determine the shape of the accessible parts of the $f_{a}(a)$ and $f_{q}(q)$ distributions, and as noted above, one has to allow for a variation with mass, or in practice, color. For the Table 1 colorintervals $\mathrm{C} 1-\mathrm{C} 7$, most of the stars are from the main sequence. For the redder intervals, there is the added difficulty that many of the TYC stars are red giants, while the double-star analysis should use only the main sequence ones. The general selection model as derived above uses complete HIP and TYC data, but for the DS analysis, an extra filtering excludes these red giants and supergiants. Because this makes for extra systematic errors, and because red MS stars are relatively few, it proved difficult to use redder selections than C9. The masses are known in the model runs, and means for the primary and the system masses are also given in Table 1 .

For each of the runs in Tables A.1 and A.2, and for each color-interval, the basic results are histogram counts of
Table 2. The total number of observed HIP binaries $(H p=6.5-10.5)$ in the five $\Delta m$ bins, in each of the nine interlaced main-sequence color bins defined in Table 1.

\begin{tabular}{lccccc}
\hline \hline color $\backslash \Delta m$ & $0-0.45$ & $0.45-0.9$ & $0.9-1.8$ & $1.8-2.7$ & $2.7-3.6$ \\
\hline C1 & 90 & 89 & 204 & 214 & 264 \\
C2 & 125 & 109 & 240 & 281 & 291 \\
C3 & 107 & 77 & 144 & 205 & 189 \\
C4 & 112 & 77 & 146 & 172 & 165 \\
C5 & 190 & 103 & 204 & 207 & 216 \\
C6 & 404 & 214 & 324 & 332 & 321 \\
C7 & 512 & 284 & 379 & 324 & 326 \\
C8 & 386 & 217 & 320 & 286 & 211 \\
C9 & 207 & 102 & 196 & 148 & 108 \\
\hline
\end{tabular}

modelled HIP stars, which are then compared with the actually observed ones. While ideally, a detailed fitting should be done in bins of galactic latitude, magnitude, separation and magnitude difference, the limited number of observed binaries made it necessary to add all magnitudes (6.5-10.5) and all galactic latitudes. The final output is thus only the marginal distributions in five logarithmic separation bins (0.3-0.6, 0.6-1.2, 1.2-2.4, 2.4-4.8 and 4.8-9.6 arcsec) and five bins of magnitude difference $(0-0.45,0.45-0.9,0.9-1.8,1.8-2.7,2.7-3.6 \mathrm{mag})$. At separations below 0.3 arcsec, the magnitude differences were deemed too poor, and at separations above some 10 arcsec, the double star identifications are incomplete and the observations also of poor quality. For the magnitude differences, the data should be almost complete to $\Delta m=3.6$, while the two smaller bins serve to show the narrowness of the $q=1$ peak.

Table 2 lists the reasonably large total HIP counts in the five $\Delta m$ bins (with similar size numbers distributed over five separation bins). The basic premise of the present investigation is that these high counts relative to more strictly complete samples represent a net gain that is not offset by poorly understood systematic errors.

\subsection{Model fits for $f_{a}$}

In all the model runs, the input $f_{a}(a)$ was the log-normal distribution in Eq. (1), but because the observed (angular) separations cover only a 1.5 dex interval, it is impossible to estimate more than one shape parameter for the $a$-distribution. In one model, I assumed that the maximum in Eq. (1) is always $\lg a_{m}=1.5$, and then found the width $\left(\sigma_{a}\right)$ giving the best fit. In an alternative model, I estimated instead the slope $s_{a}$ in a $\log$-log relation of the number of binaries versus orbit size

$\lg N=$ const. $+s_{a} \lg a$

which in its non-logarithmic form corresponds to the power-law $f_{a}(a) \sim a^{\left(s_{a}-1\right)}$. In both cases, the linear orbit-sizes $a$ are taken proportional to the observed angular separations $\alpha$, with a scaling factor given by the Galaxy model run. (Because the majority of the stars are in a small range of absolute and apparent magnitude, this should be a permissible approximation, leading to a much simplified analysis.)

The input $f_{a}^{\text {in }}(a)$ produces five model counts $N_{\text {mod }}(\alpha)$, and assuming first-order linearity, we expect that the updated value

$f_{a}^{\text {out }}(a)=f_{a}^{\text {in }}(a) N_{\text {obs }}(\alpha(a)) / N_{\text {mod }}(\alpha(a))$

would have produced the actually observed HIP count $N_{\text {obs }}(\alpha)$. Since no attempt is made to derive absolute levels of binarity, only relative errors are important, and the mean errors of the 
five (lg) $f_{a}^{\text {out }}$ points are estimated as described in Appendix B. They are then fitted in the first model to a $N^{\prime}\left(\lg a-1.5, \sigma_{a}\right)$ distribution. The best $\sigma_{a}$ is found by minimizing the 4 d.o.f. $\chi^{2}$, and the 1 -sigma confidence limit is taken at $\chi_{\min }^{2}+1$. In the alternative model, $s_{a}$ is the slope in the $\lg f_{a}^{\text {out }}-\lg a$ plot, with its mean error given by simple standard equations. Both models give useable fits to the data, see Sect. 5.1.

Although questions about the real binary fractions are fundamental, there are difficult normalization problems when using only small parts of the $f_{q}(q)$ - and $f_{a}(a)$-distributions, aggravated by the questions of how to normalize in the presence of magnitude- and color-dependent misfits of the distributions. In Söderhjelm (2000), I concluded tentatively that the binary frequencies seem appreciably higher than those given by Duquennoy \& Mayor 1991, and with the generally broader $a$-distribution derived below, this may still hold.

\subsection{Model fits for $f_{q}$}

The model to be fitted to the observable part $(q>0.5)$ of the $q$-distribution is the second part of Eq. (4)

$f_{q}(q) \sim q^{C_{q}}\left[1+C_{p} P(q)\right]$.

We have the model and observed counts at five $\Delta m$, and with a transformation to $q$ from the Galaxy model, we obtain the data to be fitted as

$f_{q}^{\text {out }}(q)=f_{q}^{\text {in }}(q) N_{\text {obs }}(\Delta m(q)) / N_{\text {mod }}(\Delta m(q))$.

Again, only the shape of the distribution is studied, and the mean errors in $\lg f_{q}^{\text {out }}$ follow from Eq. (B.1). Putting $C_{p}=0$ in Eq. (7), the simplest model is a pure power-law. The $\chi^{2}$ goodness-of-fit for such a fit is normally very poor because of an offending high point close to $q=1$, and the alternative fitting model is then the full (power-law + peak) Eq. (7). Effectively, four points give $C_{q}$, and the high- $q$ point determines the peak amplitude $C_{p}$. The fit has to be done iteratively, since the next-highest $q$-point may be affected by the peak, but there are generally no convergence problems, and we get $C_{q}$ and $C_{p}$ together with their estimated mean errors.

Obviously, as a single point value, the derived $C_{p}$ depends strongly on the assumed width of the peak. From experiment, the best-fit width may be as low as 0.03 at the lowest-mass bins (where the luminosity varies most steeply with mass and hence the $q$-resolution is best), but with a possible increase at higher masses. The present data are insufficient to settle this question, and in the end I used the compromise width 0.04 at all masses.

\subsection{Combined results}

Each of the model-runs gives one or two parameters (with estimated mean errors) per $f_{a} / f_{q}$ model and per color-bin, and as the final result of the present investigation, we derive a weighted mean $P_{m}$ for each parameter in each color-bin. The results are not sensitive to the weighting, but using the inverse $3 / 2$ power of the individual mean errors instead of the standard inverse square diminishes any potential problems from mean errors based on the ad hoc Eq. (B.1). Individual parameter values $P_{i}$ deviating more than about $3 \sigma$ from the mean were also iteratively removed, allowing for different numbers of included runs $\left(n_{r}\right)$ for different parameters and different colors. The deviations from the mean give a normalized $\chi^{2}$ goodness-of-fit

$G_{n}=\Sigma\left(\frac{P_{i}-P_{m}}{\sigma_{i}}\right)^{2} /\left(n_{r}-1\right)$
Table 3. The mean width $\left(\sigma_{a}\right)$ and the alternative slope $\left(s_{a}\right)$ of $f_{a}(\lg a)$, as computed from data in nine interlaced color bins. In both cases, the mean errors in these final parameter values are computed from their inter-run spreads. The second column gives the mean $\lg a[\mathrm{au}]$ in the first and last separation-bins, and in the last two columns, the $\chi^{2}$ significance $\lg Q_{P}$ and the ratio $R_{Q a}$ (see text) are listed.

\begin{tabular}{cccccccc}
\hline \hline bin & $\lg a[\mathrm{au}]$ & $\sigma_{a}$ & m.e. & $s_{a}$ & m.e. & $\lg Q_{P}$ & $R_{Q a}$ \\
\hline C1 & $2.18-3.37$ & 2.74 & 0.38 & -0.020 & 0.016 & -1.1 & -0.7 \\
C2 & $2.14-3.34$ & 2.49 & 0.15 & -0.073 & 0.009 & -1.5 & -0.3 \\
C3 & $2.07-3.26$ & 1.66 & 0.06 & -0.167 & 0.011 & -0.8 & -0.3 \\
C4 & $2.00-3.19$ & 1.75 & 0.05 & -0.149 & 0.009 & -1.2 & -0.1 \\
C5 & $1.93-3.10$ & 1.84 & 0.05 & -0.116 & 0.009 & -0.7 & 0.0 \\
C6 & $1.82-3.00$ & 1.63 & 0.07 & -0.130 & 0.014 & -0.9 & 0.3 \\
C7 & $1.74-2.92$ & 1.49 & 0.05 & -0.149 & 0.011 & -1.4 & 0.4 \\
C8 & $1.67-2.85$ & 1.42 & 0.03 & -0.154 & 0.007 & -0.8 & 0.3 \\
C9 & $1.58-2.77$ & 1.13 & 0.03 & -0.191 & 0.010 & -0.5 & 0.1 \\
\hline
\end{tabular}

which through the adjusted $A_{w}$ in Eq. (B.1) is generally of the order of unity. Variations of $G_{n}$ with color or between different parameters may however be studied, see Fig. 8.

It is not trivial to estimate the final mean errors in the parameters, but from the Monte Carlo experiments suggesting the $3 / 2$ power weighting, I derived the approximation

$\sigma\left(P_{m}\right)=\left(\frac{\Sigma\left(P_{i}-P_{m}\right)^{2}}{n_{r}\left(n_{r}-1.75\right)}\right)^{1 / 2}$

which has been used to calculate the final mean errors in Tables 3 and 4.

\section{Double star distribution results}

\section{1. $f_{a}$ parameters}

As described in Sect. 4.1, one makes for each run in Tables A.1 and A.2, and for each color-bin, a fit either to a normal (N) or to a power-law (P) model for $f_{a}(a)$. The $\chi^{2}$ goodness-of-fits can be translated to probabilities $Q$ that the $\chi^{2}$ for 5-2 d.o.f. is as high as the observed one. The logarithmic means of these $Q$-values $\left(Q_{N}\right.$ and $\left.Q_{P}\right)$ are not too small, and because the input errors from Eq. (B.1) are somewhat ad hoc, both models are useable. Because they both use the same input sigmas, the ratio $R_{Q a}=\lg \left(Q_{N} / Q_{P}\right)$ gives some grading of the models. As seen in the last column of Table 3 , there is a slight preference for the power-law at high masses, and for the normal distribution at lower masses, but still we may use both. The other columns and Fig. 2 show the final means and their estimated mean errors for $\sigma_{a}$ and $s_{a}$, and even if $\sigma_{a}$ is close to the standard Duquennoy $\&$ Mayor (1991) value around 1.5, the variations with mass are clearly significant. All the present fittings are on the wide side of the $f_{a}(a)$ maximum, and the downslope towards large separations clearly diminishes with increasing mass. Above a system mass around 6 Msun, the observed $\log / \log$ slope $s_{a}$ reaches zero, equivalent to the often used "Öpik distribution" $f_{a}(a) \sim 1 / a$.

\section{2. $f_{q}$ parameters}

The five $f_{q}$ points were fitted either to a single power-law (P) or to a 4-point power-law plus a narrow peak at $q=1(\mathrm{PP})$. As for the two $f_{a}$-models, the mean $Q_{P}$ and $Q_{P P}$ probabilities were derived, but in this case, the model with a peak showed a much better fit. In Table 4, therefore, only the PP $C_{q}$ and $C_{p}$ values are given, together with their mean errors. The last columns 

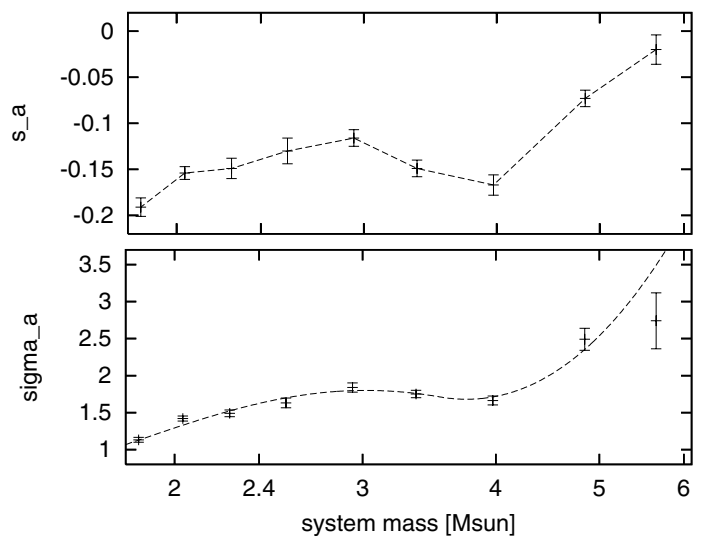

Fig. 2. The upper panel shows the $f_{a}(\lg a)$ slope $s_{a}$, and the lower panel the width $\sigma_{a}$ of a fitted log-normal distribution. The trends are very equal, with a shallower slope/broader distribution at larger system masses. The smooth curve in the lower panel is the analytical approximation used in run B218.

Table 4. The mean power-law exponent $\left(C_{q}\right)$ and peak amplitude $\left(C_{p}\right)$ parameters, with their observed mean errors, for a power-law plus peak (PP) model of $f_{q}(q)$ according to Eq. (7). The fits are done for a limited range of $q$-values, as shown in Col. 2. The last column gives the mean ratios $\lg \left(Q_{P P} / Q_{P}\right)$, positive values indicating that the PP model fits (much) better than the 5-point power-law $(\mathrm{P})$ one.

\begin{tabular}{rrrrrrrl}
\hline \hline bin & $q$-interval & $C_{q}$ & m.e. & $C_{p}$ & m.e. & $\lg Q_{P P}$ & $R_{Q q}$ \\
\hline C1 & $0.47-0.95$ & -2.00 & 0.07 & 0.44 & 0.12 & -0.8 & 0.2 \\
C2 & $0.50-0.96$ & -1.88 & 0.05 & 0.51 & 0.07 & -0.6 & 0.8 \\
C3 & $0.54-0.96$ & -2.05 & 0.06 & 1.10 & 0.08 & -1.6 & 3.8 \\
C4 & $0.57-0.96$ & -1.80 & 0.06 & 0.90 & 0.07 & -0.9 & 3.2 \\
C5 & $0.58-0.96$ & -1.96 & 0.07 & 1.30 & 0.09 & -0.3 & 5.1 \\
C6 & $0.60-0.97$ & -1.78 & 0.09 & 1.31 & 0.10 & -0.6 & 6.6 \\
C7 & $0.63-0.97$ & -1.04 & 0.11 & 1.19 & 0.06 & -1.1 & 6.1 \\
C8 & $0.65-0.97$ & +0.34 & 0.13 & 1.00 & 0.08 & -0.5 & 3.3 \\
C9 & $0.66-0.97$ & +0.55 & 0.17 & 1.20 & 0.13 & -0.8 & 1.7 \\
\hline
\end{tabular}

list $\lg Q_{P P}$ and the ratio $R_{Q q}=\lg Q_{P P}-\lg Q_{P}$. A value 6 indicates (formally) a $10^{6}$ times higher probability for the model with a peak versus one without, and only for the highest masses the peak may be absent. The $C_{q}$ and $C_{p}$ values are also shown in Fig. 3, together with the analytical interpolation curves used in run $\mathrm{B} 218$.

Apart from the presence of a $q=1$ peak in the massinterval covered, the most noteworthy feature of Fig. 3 is the rapid change of the $C_{q}$ exponent between system masses 2.0 and 2.5 Msun. Although this transition in reality must be more gradual, the major change in the $f_{q}(q)$ slope is likely a real feature. As explained above, the normalization is very complicated, and in practice, I did not use any absolute levels and fitted only relative to a mean over the five $q$-points. Figure 4 shows typical $f_{q}(q)$-curves at equal logarithmic mass-steps, and the radical change between 2.0 and 2.5 Msun is again apparent. Note also (Table 4) that the lowest $q$-value used in the fits is only about 0.65 for the 2 Msun curve, and thus that a very large part of $f_{q}(q)$ is undefined from the present data. This means also that a "twin fraction" can not be specified, since it will depend largely on these low- $q$ parts of the distribution (For most mass-ratio distributions, the fraction of systems with $q>0.95$ will not be significantly above the simplistic estimate of $5 \%$ ).

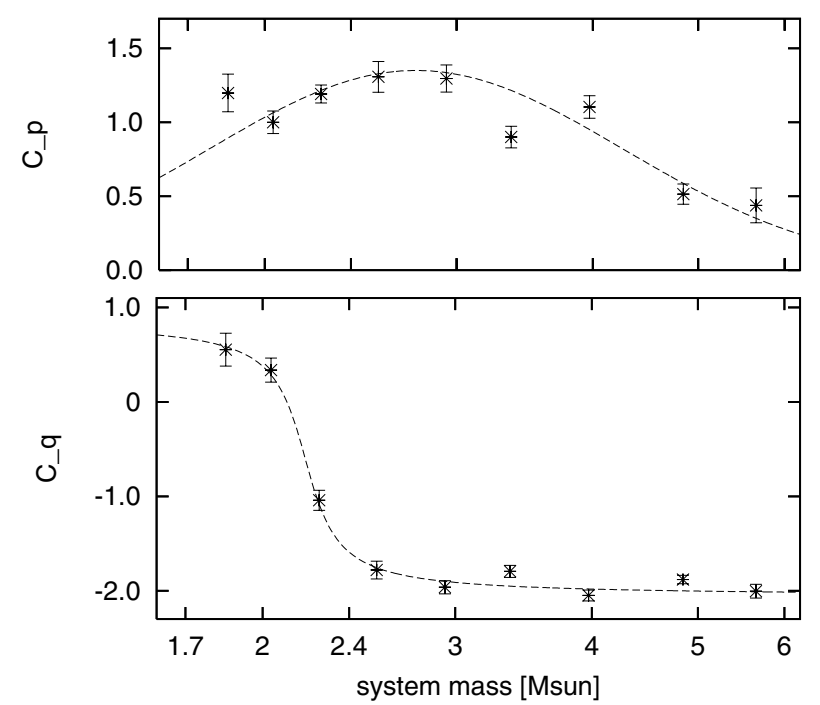

Fig. 3. In the lower part of the figure are shown the values for the $C_{q}$ exponent and in the upper part the $C_{p}$ peak amplitudes, for the Eq. (7) fits to $f_{q}(q)$. The nine color-bins correspond to nine values of the mean system mass of the binaries, and the dashed lines show the ad hoc analytical fits used in run B218.

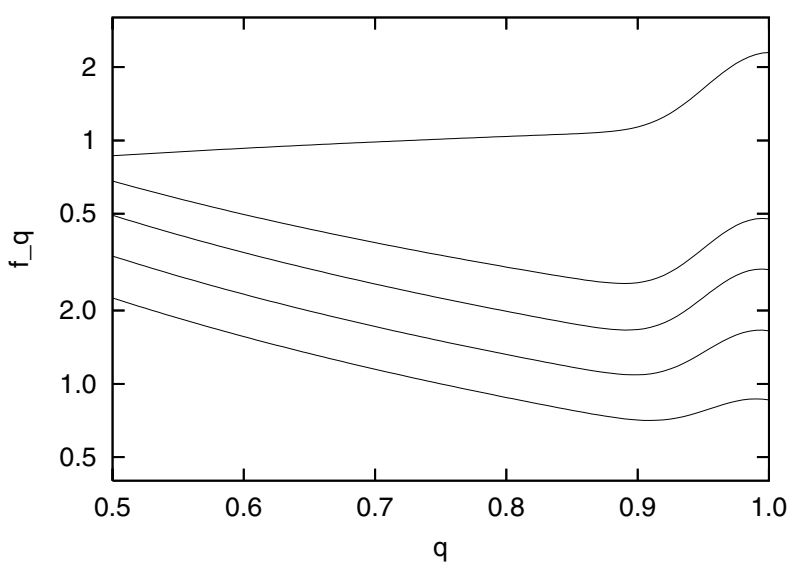

Fig. 4. Typical $f_{q}(q)$-curves at system masses 2.0 Msun (top), 2.5, 3.2, 4.0 and 5.0 Msun (bottom), using the smooth curves in Figs. 2 and 3. The logarithmic $y$-scale is indicated for the top and bottom curves.

\subsection{The B218 run}

For the last (B218) model Galaxy run, the input parameters for $f_{q}(q)$ were taken as the smooth curves close to the final datapoints shown in Figs. 2 and 3. Therefore, ideally, one expects the uncorrected obs/model results to be close to unity in all color/sep/ $\Delta m$ bins. For various reasons, this is not realized in practice. Firstly, because of the poor blue-star modelling, even a large run like B218 sometimes has below 100 model binaries in a separation or $\Delta m$ bin, with correspondingly large counting uncertainties. More importantly, however, since the fittings are done relative to the irregularly sampled HIP data, the best obs/model plots would only be obtained with inputs close to the results shown in Fig. 3/Table 4. With input parameters from a smooth model, we have extra misfits due to the peculiarities of the Hipparcos Input Catalog. In Fig. 5, it is thus difficult to see any major difference between run B218, with a massdependent $\sigma_{a}$, and run B58, with a constant but reasonable value. In Fig. 6, it is more apparent that the B218 run is a definite improvement relative to the B170 run, which assumed a zero 

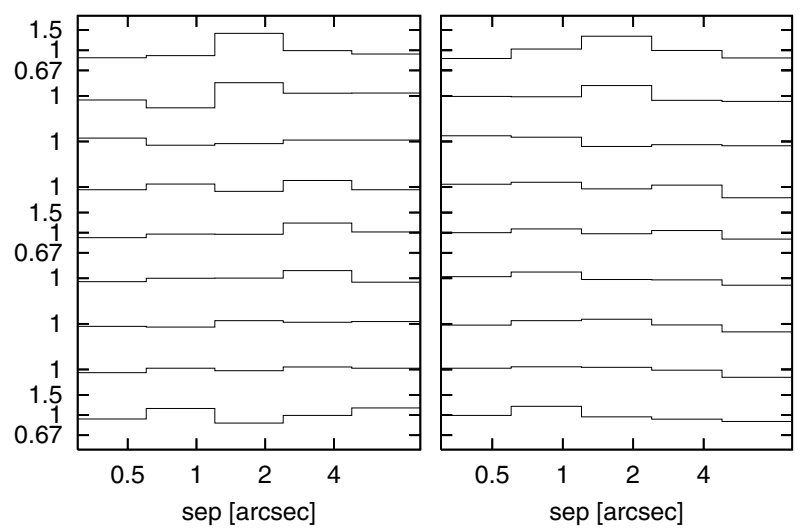

Fig. 5. The raw HIP/model count histograms with respect to (angular) separation. In the left panel are the data for the B218 run, and to the right are the data for run B58. The color bins go from $\mathrm{C} 1$ at the top to $\mathrm{C} 9$ at the bottom, and the logarithmic $y$-scale is indicated for $\mathrm{C} 1, \mathrm{C} 5$ and $\mathrm{C} 9$.

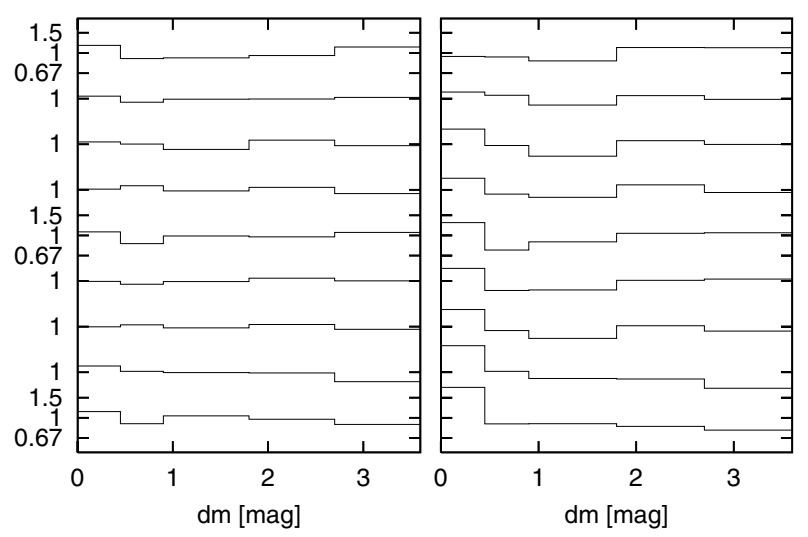

Fig. 6. The raw HIP/model count histograms with respect to magnitude difference. In the left panel are the data for the B218 run, with a good $f_{q}(q)$ input approximation, and on the right are the data for run $\mathrm{B} 70$ with no $q=1$ peak. The color bins go from $\mathrm{C} 1$ at the top to $\mathrm{C} 9$ at the bottom, and the logarithmic $y$-scale is indicated for $\mathrm{C} 1, \mathrm{C} 5$ and $\mathrm{C}$.

amplitude for the $q=1$ peak and consequently shows large systematic excesses of small- $\Delta m$ pairs.

\section{Possible systematic errors}

Although the above analysis indicates an obvious $q=1$ peak for most masses, it is a disturbing fact that it is both mass-dependent and similar to the spurious peaks discussed e.g. by Hogeveen (1990). In the present section, I try to show that implausibly large bias effects in the data are needed in order to produce the observed peak.

Systematic effects in the Hipparcos data reductions can be safely neglected. The double star analysis for Hipparcos was done independently (and with very different methods) by the NDAC and FAST reduction consortia (cf. HIP, Vol. 3), and only at a late stage combined to give the final data. One could e.g. suspect that small magnitude differences sometimes had different signs in NDAC and FAST, and that the combination produced too many near zero results. This is ruled out by a comparison of the original NDAC $\Delta m$ :s (preserved by the author) and the final HIP ones, where the (very small) difference is in the reverse sense, with the NDAC data slightly more peaked towards zero. Also, in the comparison presented in Fig. 22.7 in Vol. 3 of HIP, no systematic errors relative to ground-based CCD measurements can be seen.

In Söderhjelm (2000), I introduced a 'double-star biasfactor' $D_{b}$ to simulate an excess $\left(D_{b}>1\right)$ or a deficit of binaries relative to singles in the Hipparcos Input Catalog. I showed also that in the present sort of modellings, the data can be fitted equally well with any $D_{b}$ (a high value offset by a low duplicity fraction and vice versa), making it even harder to use the Hipparcos data to derive binary frequencies. In the present work, I have extended the bias-factor to depend also on the magnitudedifference in the pair.

This includes first the classical Öpik (1923) bias, creating an overabundance of small- $\Delta m$ systems in a magnitude-limited sample. The volume sampled increases with the combined magnitude of the pair, resulting in a function

$D_{b}(\Delta m)=\left(1+10^{-0.4 \Delta m}\right)^{1.5}$

The present analysis method, always comparing model/observed data, should be unaffected by the effect, but to be sure, I included the Eq. (11) bias in my Hipparcos selection models. Using the standard fitting models, the $C_{p}$ peak amplitudes were decreased very marginally, probably because the Öpik effect produces only a much broader peak around $\Delta m=0$, which mainly changes the $C_{q}$ slope. A similar conclusion was reached by Tokovinin (2000), testing the reality of a similarly narrow $q=1$ peak for spectroscopic binaries, cf. Sect. 7.

As shown e.g. by Hogeveen (1990), there is a particularly heavy bias towards equal-magnitude systems in the Fourth Catalog of Orbits of Visual Binary Stars (Worley \& Heintz 1983), which was the last available in the preparation of the Hipparcos Input Catalog. Many of the binaries in that cata$\log$ were high-priority objects for parallax determination by Hipparcos, and it is conceivable that this has produced a peaked selection, with small- $\Delta m$ systems more likely to be included. This has been modelled by an ad hoc Gaussian model

$D_{b}(\Delta m)=1+B_{\Delta m} N^{\prime}\left(\Delta m, \sigma_{b}\right)$

where the amplitude and width of the bias can be freely selected.

Using the Eq. (12) bias with $\sigma_{b}=0.3$, I tried a number of different $B_{\Delta m}$ amplitudes, and going through the Eq. (7) fits again, one has a very obvious trend with smaller $C_{p}$ peaks as the bias $B_{\Delta m}$ increases. Figure 7 shows some sample results, and since the goodness-of-fit is almost unchanged (Fig. 8), it is not possible to rule out this special kind of bias using only the present modellings. Changing the width and/or shape of the bias-peak changes the details, but not the main conclusions.

From Fig. 7, it is clear however that to reduce $C_{p}$ to zero, one needs an $B_{\Delta m}$ around 2, that is, a binary with $\Delta m=0$ has to be 3 times as likely to be included in HIP than a system with $\Delta m>=1$, which a priori seems quite improbable. But Fig. 7 shows also that a more plausible bias $\left(B_{\Delta m}=0.25-0.5\right)$ reduces $C_{p}$ appreciably, and this is harder to rule out.

By its more rigorous sampling criteria, the study by Söderhjelm (1997) should place further constraints. The $f_{q}(q)$ data in Table 4 and Fig. 5 in that investigation can be fitted to the present Eq. (7) model, giving $C_{q}$ and $C_{p}$ values for samples with different absolute magnitudes and different linear separations. Although in 1997 I tried to show an $a$-dependence in $f_{q}(q)$, it may alternatively be viewed as a mass-dependence, and I have combined the three "F" $(V-I=0.4-0.7)$ and the three "A" $(V-I=0.0-0.4)$ samples to the data in Table 5. Because of the originally small number of systems, the mean errors of the means in Table 5 (derived from an application of Eq. (10)) are 


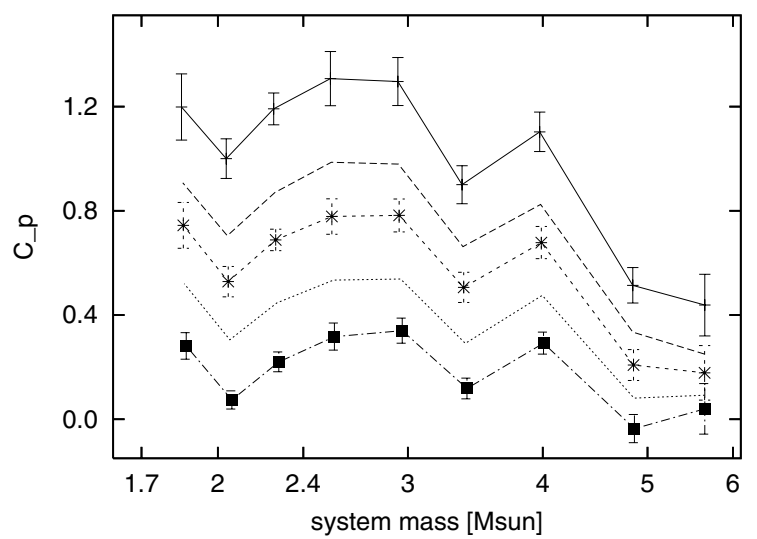

Fig. 7. The $C_{p}$ peak amplitudes for the Eq. (7) fits to $f_{q}(q)$, as derived with various selection biases according to Eq. (12). The uppermost curve is for the unbiased standard solution $\left(B_{\Delta m}=0\right)$, and then follow $B_{\Delta m}=0.25,0.5,1.0$ and 2.0 (lowest curve).

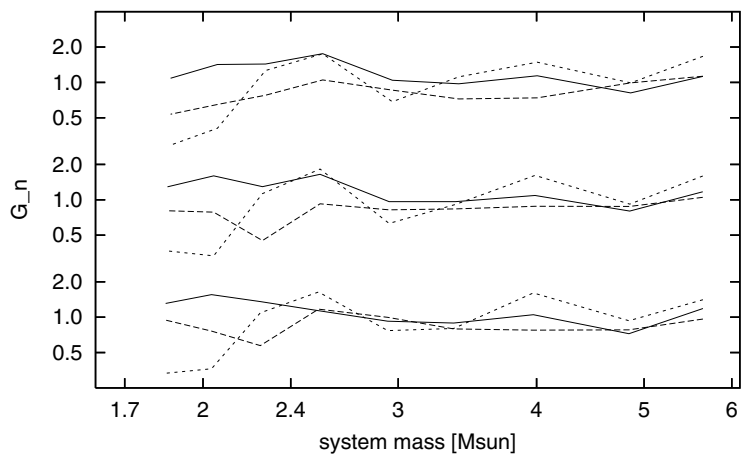

Fig. 8. The $G_{n}$ (Eq. (9)) goodness-of-fit values for $C_{q}$ (full lines), $C_{p}$ (dashed lines) and $s_{a}$ (dotted lines), for the unbiased run (bottom), the biased one with $B_{\Delta m}=0.5$ (middle) and the one with $B_{\Delta m}=2.0$ (top).

Table 5. The mean $f_{q}(q)$ histograms for an "F" ( $\left.V-I=0.4-0.7\right)$ and an "A" $(V-I=0.0-0.4)$ group of binaries, from the paper by Söderhjelm (1997).

\begin{tabular}{rrrrr}
\hline \hline$q$-interval & $f_{q}(A)$ & m.e. & $f_{q}(F)$ & m.e. \\
\hline $0.95-1.00$ & 0.33 & 0.048 & 0.63 & 0.066 \\
$0.90-0.95$ & 0.25 & 0.038 & 0.40 & 0.052 \\
$0.80-0.90$ & 0.20 & 0.030 & 0.27 & 0.030 \\
$0.70-0.80$ & 0.30 & 0.036 & 0.33 & 0.034 \\
$0.60-0.70$ & 0.42 & 0.040 & 0.36 & 0.032 \\
$0.55-0.60$ & 0.44 & 0.036 & - & - \\
\hline
\end{tabular}

large, and the parameters derived are of low precision. For the A sample we get $C_{q}=-1.75(0.30), C_{p}=0.97(0.42)$, and for the F sample $C_{q}=-0.64(0.40), C_{p}=1.57(0.44)$, mean errors in parentheses. Still, if the 1997 data can be trusted, these peak amplitudes are of a size that more or less rule out a very large $\Delta m$-bias. The $C_{q}$ value for the $\mathrm{F}$ sample is large, but placing the above $C_{p}$ values in Fig. 7, one may derive a probable limit $B_{\Delta m}<0.5$, and a $q=1$ peak in $f_{q}(q)$ should be present in the whole mass-interval covered in this study.

The rapid change of the $C_{q}$ exponent with mass shown in Fig. 3 is almost unchanged by an Eq. (12) bias, Fig. 9 showing a rather larger discontinuity with a $B_{\Delta m}=2$ bias amplitude. Although other kinds of bias might have a larger influence, the change of slope seems to be a robust result.

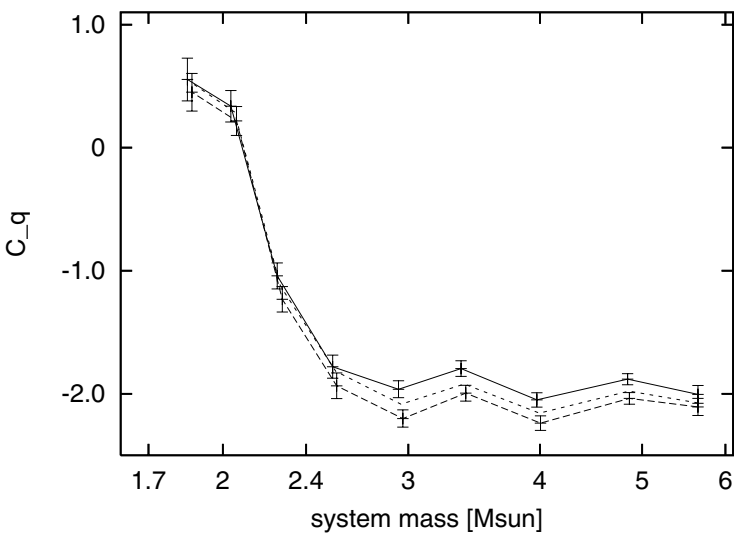

Fig. 9. The power-law slope $C_{q}$ as derived with no bias (full line) and with Eq. (12) selection bias: $B_{\Delta m}=1.0$ (dotted line) and $B_{\Delta m}=2.0$ (dashed line).

\section{Comparison with earlier studies}

As shown e.g. in the study by Hogeveen (1990), different observational sampling effects may give vastly different observed $f_{q}(q)$-distributions. To get around such problems, most modern studies tend to use small, almost complete samples, where these selection effects can be controlled and corrected for. Most studies of the mass-ratio distribution are also geared towards reaching the interesting small- $q$ part, but the combination of small sample size and a wide range in $q$ makes it impossible to discern any details in the distribution around $q=1$.

A case in point is the classical Duquennoy \& Mayor (1991) G-star study, which used a total of only 18 visual binaries with $q>0.5$. A peak of the kind derived in the present paper is obviously lost in the noise. In a study by Eggenberger et al. (2004) using Hipparcos data, the authors do see a $q=1$ peak but discard it as a statistical artifact. Similar problems with low-number statistics can be seen in the investigations of spectroscopic systems by Goldberg et al. (2003), Mazeh et al. (2003) or Halbwachs et al. (2003), and there is no general agreement as to the presence and/or width of a $q=1$ peak, or its relation to the orbital period of the systems.

Some recent wide binary studies use high-resolution imaging in nearby clusters or associations. In the sample of low-mass $\mathrm{T}$ Tauri binaries observed by Woitas et al. (2001), the problem lies in translating magnitude to mass, and different sets of stellar models give different mass-ratio distributions. Again the analysis is done with $q$-bins of width 0.2 , and a narrow $q=1$ peak would be undetectable. For B stars in Sco OB2, Shatsky \& Tokovinin (2002) have only 13 stars with $q>0.4$, but the distribution decreases towards $q=1$. In the more extensive study of the A stars in the same association, Kouwenhoven et al. (2005) give (in their Fig. 14) a very interesting $f_{q}(q)$ histogram, showing both a rapid decrease from $q=0.5$ to 0.9 and a peak at $q=1$, closely resembling the results of the present study. Again, however, this is based on only 25 stars with $q>0.5$, and the agreement may be fortuitous.

In an important study with a high-resolution sampling around $q=1$, Tokovinin (2000) found a convincing peak for low-mass binaries with periods shorter than some 40 days. The problem is still that there are so few SB2 orbits available for periods above 40 days that the only certain conclusion would be that the peak is smaller there, not necessarily absent. Lucy (2006), using SB2:s with no discrimination as to period, finds a significant and very narrow $q=1$ peak (Gaussian width around 0.02). 
However he also discards the study by Pinsonneault \& Stanek (2006), who claim an overabundance of twins for eclipsing binaries in the SMC.

For the orbit-size distribution, Poveda \& Allen (2004) have long maintained that many binaries of the typical sizes used here follow the scale-free Öpik distribution, which then steepens only for wider and older pairs. With $f_{a}(a) \sim 1 / a^{\left(1+s_{a}\right)}$, and noting the smallness of all the $s_{a}$ values in Table 3 , there is actually little contradiction. A steeper downslope for less massive systems may be due to smaller tidal limits in the field of the Galaxy, but the dominant cause probably lies in the binary formation processes.

\section{Conclusions and directions for future studies}

The most important conclusion of the present study is that an excess of pairs with very similar component masses is seen in the Hipparcos sample of very wide (50-1000 au) binaries. This is a hard-to-understand fact that has to be related to their formation processes. The fraction of twins may be low, but it is not zero, and some of these binaries must have been formed as such, and not as the result of dynamical processes coupling unrelated objects. Although many of the observed characteristics of $f_{q}(q)$ may be well reproduced from such dynamical models (cf. Hubber \& Whitworth 2005; or Valtonen 2004), a $q=1$ peak must have some other explanation.

In Valtonens study, one has to have a separate mechanism for producing short-period ( $<10 \mathrm{yr}$ ) pairs. One such model (Bonnell $\&$ Bate 2005) uses accretion to transform wide, lower-mass, unequal- $q$ pairs into higher-mass, short-period twins, and this could explain e.g. the (tentative) Pinsonneult \& Stanek (2006) results. Generally, for these accretion models (Bate 2000), closer and more massive binaries are more likely to form twins, and one way to reconcile this prediction with the present evidence would be to return to the Söderhjelm (1997) interpretation of the massdependence as a dependence on the separations. The Hipparcos binaries are however all so wide that an accretion-driven evolution towards $q=1$ seems unlikely.

The presence of a $q=1$ peak for wide binaries remains thus a mystery. Also, although a variation of the slope of $f_{q}(q)$ with binary system mass is natural, the Hipparcos data show a surprisingly rapid change over a short mass-interval. The simplest explanation is to regard both these results as spurious, to be traced back to the Hipparcos Input Catalog, where the complex selection process has resulted in distributions with too many hardto-model details. Speaking against such an interpretation is the evidence from Fig. 9 that the change-of-slope is a more robust result, at least with respect to $\Delta m$-bias. It is obviously important to check the results using other sources of data.

Although the imaging data from the Sloan Survey (SDSS), cf. e.g. Gunn et al. (1998) or Adelman-McCarthy et al. (2006), have mainly been used for other purposes, it could be a place to check for twins in wide binaries. There could be a small separation-interval between the resolution-limit at around 3 arcsec and (say) 10 arcsec (where optical pairs start to dominate), in which one could try to identify potential physical binaries. Approximate absolute magnitudes could be derived from the 5-color photometry, and for the promising cases, one would then also have estimates of the masses, the linear orbit size and the mass-ratio (from $\Delta m$ ). With 100 million stars to start from, it should be possible to obtain a sample large enough to definitely confirm or refute the Hipparcos result.

If this plan fails (e.g. because the limited angular resolution produces a too strong observational selection-dependence
Table A.1. List of model galaxy runs using Eq. (2) for the mass-ratio distribution. The age column gives the age-interval in Gyr, and $f_{\mathrm{c}}$ is the coverage normalized to nominal all-sky star-counts. The rest of the table gives the input parameters in $f_{a}$ and $f_{q}$.

\begin{tabular}{lccccccccc}
\hline \hline id & age & $f_{\mathrm{c}}$ & $\lg a_{m}$ & $\sigma_{a}$ & $A_{0}$ & $A_{q}$ & $q_{m}$ & $\sigma_{q}$ & $A_{p}$ \\
\hline 45 & $0-12$ & 1.0 & 1.5 & 1.8 & 1 & 2 & 0.2 & 0.30 & 1.50 \\
68 & $0-12$ & 1.0 & 1.5 & 2.0 & 1 & 0 & - & - & 1.50 \\
10 & $0-12$ & 1.0 & 1.5 & 1.8 & 0 & 1 & 0.6 & 0.40 & 1.00 \\
11 & $0-12$ & 1.0 & 1.5 & 1.8 & 0 & 1 & 0.6 & 0.30 & 1.00 \\
123 & $0-12$ & 1.0 & 1.5 & 1.8 & 0 & 1 & 0.6 & 0.25 & 0.75 \\
145 & $0-12$ & 1.0 & 1.5 & 1.8 & 0 & 1 & 0.4 & 0.35 & 0.80 \\
168 & $0-12$ & 1.5 & 1.5 & 1.8 & 0 & 1 & 0.4 & 0.35 & 0.80 \\
191 & $0-12$ & 1.5 & 1.5 & 1.8 & 0 & 1 & 0.4 & 0.35 & 0.70 \\
224 & $0-12$ & 1.5 & 1.5 & 1.8 & 0 & 1 & 0.4 & 0.35 & 0.40 \\
B01 & $0-2$ & 3.0 & 1.5 & 1.8 & 0 & 1 & 0.6 & 0.25 & 0.40 \\
B02 & $0-2$ & 3.0 & 1.8 & 1.3 & 0 & 1 & 0.7 & 0.20 & 0.40 \\
\hline
\end{tabular}

Table A.2. List of model galaxy runs using Eqs. (3) or (4) for the massratio distribution. The first columns are as in Table 1, and then follow the alternative $f_{q}(q)$ parameters. In the last four runs, the parameters varied with mass.

\begin{tabular}{lcccccccc}
\hline \hline id & age & $f_{\mathrm{c}}$ & $\lg a_{m}$ & $\sigma_{a}$ & $B_{q}$ & $B_{p}$ & $C_{q}$ & $C_{p}$ \\
\hline B03 & $0-3$ & 2.0 & 2.0 & 1.6 & 1.82 & 0.36 & & \\
B04 & $0-4$ & 1.5 & 2.0 & 1.6 & 0.00 & 0.00 & & \\
B58 & $0-12$ & 4.0 & 1.5 & 1.8 & 1.60 & 0.60 & & \\
B92 & $0-12$ & 4.0 & 1.5 & var. & var. & var. & & \\
B136 & $0-12$ & 4.0 & 1.5 & var. & & & var. & var. \\
B170 & $0-12$ & 4.0 & 1.5 & var. & & & var. & 0.00 \\
B218 & $0-12$ & 2.0 & 1.5 & var. & & & var. & var. \\
\hline
\end{tabular}

on $\Delta m$ ), an even simpler method may be to just cross-check SDSS with existing lists of wide binaries. The defining feature of the Hipparcos peak is its narrowness, which means that an Öpik-like bias is not very dangerous. Care would be needed to quantify it, but the presence or otherwise of a $q=1$ peak should be clear.

Noting finally the interesting results from Sco OB2 mentioned above (Sect. 7), it seems clear that the accumulated data from similar direct studies will soon include enough systems to enable a significant test for the reality of a $q=1$ peak for wide binaries.

Acknowledgements. I thank a second referee for interesting references and valuable hints on how to make this paper more readable

\section{Appendix A: Galaxy model runs}

Tables A.1 and A.2 give the list of Galaxy model runs used in this study, with input parameters for $f_{a}$ and $f_{q}$ as defined in Eqs. (1)-(4). The unsystematic parameter choices reflect some major problems in the evolving analysis programs, but even the "unrealistic" runs has contributed to and strengthened the final results. A run as given in these tables was mostly assembled from several parts $(168=16+17+18, \mathrm{~B} 218=\mathrm{B} 21-\mathrm{B} 28)$, differing in sky-coverage or age-distributions or both. Experiments with a time-varying star formation rate did not prove fruitful, and the combined runs correspond to a roughly uniform SFR for ages 0-12 Gyr (except for some "B" runs that cover only younger ages). 


\section{Appendix B: Assumed errors in the updated $f_{a}$ and $f_{q}$ points}

The relative error of a $f_{a}^{\text {out }}$ or $f_{q}^{\text {out }}$ value is seen from Eqs. (6) and (8) to be given mainly by the relative error in $N_{\text {mod }}$. It can be thought of also as the mean error $\left(\sigma_{u}\right)$ in $\ln f_{a}^{\text {out }}$ or $\ln f_{q}^{\text {out }}$, and it is in reality a complex mix of (Galaxy) model errors and observational (selection model) effects. A lower limit to $\sigma_{u}$ should however be given simply by the counting error $N_{\mathrm{mod}}^{-0.5}$. Here, $N_{\text {mod }}=f_{\mathrm{c}} N_{\mathrm{s}}$ is the number of actually counted (model) stars in a bin, with $N_{\mathrm{s}}$ the nominal (all-sky) count, and $f_{\mathrm{c}}$ the size of the model run. (Normally $f_{\mathrm{c}}>1$ in order to increase the model star numbers and decrease the random errors). These minimum $\sigma_{u}$ give errors in the fitted $f_{a}(a)$ and $f_{q}(q)$ parameters that are too small when compared with the (internal) scatter between the different Galaxy modellings. Therefore, I arbitrarily added an extra term (with a free parameter $A_{w}$ ) in the expression for the assumed variance

$\sigma_{u}^{2}=\left(1+A_{w} f_{\mathrm{c}}\right) /\left(f_{\mathrm{c}} N_{\mathrm{s}}\right)$

reflecting the fact that increasing $f_{\mathrm{c}}$ arbitrarily should not lead to a zero mean error. Comparing the between-runs $\chi^{2}$ goodnessof-fit (Eq. (9)) for a number of different assumed $A_{w}$, a value around 0.5 seemed realistic. Noting that the extra variance seems to be present mostly in the redder bins, I also introduced a colordependence, putting $A_{w}=\langle V-I\rangle^{2}$, where $\langle V-I\rangle$ is the mean color in the analyzed bin. A simple final scaling gives the mean errors in $\lg f$ instead of $\ln f$.

\section{References}

Adelman-McCarthy, J. K., Agüeros, M. A., Allam, S. S., et al. 2006, ApJS, 162, 38

Bate, M. R. 2000, MNRAS, 314, 33
Bonnell, I. A., \& Bate, M. R. 2005, MNRAS, 362, 915

Duquennoy, A., \& Mayor, M. 1991, A\&A, 248, 485

ESA 1997, The Hipparcos and Tycho Catalogues, ESA SP-1200

Eggenberger, A., Halbwachs, J.-L., Udry, S., \& Mayor, M. 2004, Rev. Mex. Astron. Astrofis. Ser. Conf., 21, 28

Eggleton, P. P., Fitchett, M. J., \& Tout, C. A. 1989, ApJ 347, 998

Flower, P. J. 1996, ApJ, 469, 355

Goldberg, D., Mazeh, T., \& Latham, D. W. 2003, ApJ, 591, 397

Gunn, J. E., Carr, M., Rockosi, C., et al. 1998, AJ, 116, 3040

Halbwachs, J. L., Mayor, M., Udry, S., \& Arenou, F. 2003, A\&A, 397, 159

Høg, E., Fabricius, C., Makarov, V. V., et al. 2000, A\&A, 355, L19

Hogeveen, S. J. 1990, Ap\&SS, 173, 315

Hubber, D. A., \& Whitworth, A. P. 2005, A\&A, 437, 113

Hurley, J. R., Pols, O. R., \& Tout, C. A. 2000, MNRAS, 315, 543

Hurley, J. R., Tout, C. A., \& Pols, O. R. 2002, MNRAS, 329, 897

Kouwenhoven, M. B. N., Brown, A. G. A., Zinnecker, H., Kaper, L., \& Portegies Zwart, S. F. 2005, A\&A, 430, 137

Kouwenhoven, M. B. N. 2006, thesis

Kouwenhoven, M. B. N., Brown, A. G. A., Portegies Zwart, S. F., \& Kaper, L. 2007, A\&A, submitted

Kroupa, P. 2001, MNRAS, 322, 231

Lucy, L. B. 2006, A\&A, 457, 629

Mazeh, T., Simon, M., Prato, L., Markus, B., \& Zucker, S. 2003, ApJ, 599, 1344 Mignard, F. 2000, A\&A, 354, 522

Nordström, B., Mayor, M., Andersen, J., et al. 2004, A\&A, 418, 989

Öpik, E. 1923, Publ. Obs. Astr. Univ. Tartu 25, No. 6

Pinsonneault, M. H., \& Stanek, K. Z. 2006, ApJ, 639, L67

Poveda, A., \& Allen, C. 2004, Rev. Mex. Astron. Astrofis. Ser. Conf., 21, 49

Shatsky, N., \& Tokovinin, A. 2002, A\&A, 382, 92

Söderhjelm, S. 1997, in Proc. Visual double stars: Formation, dynamics and evolutionary tracks, ed. J. A. Docobo, A. Elipe, \& H. McAlister, ASSL, 223, 497

Söderhjelm, S. 2000, AN, 321, 165

Söderhjelm, S. 2005, in Proc. The three-dimensional Universe with Gaia, ed. C. Turon, K. S. O'Flaherty, \& M. A. C. Perryman, ESA SP-576, 97

Tokovinin, A. A. 2000, A\&A, 360, 997

Valtonen, M. 2004, Rev. Mex. Astron. Astrofis. Ser. Conf., 21, 147

Woitas, J., Leinert, Ch., \& Köhler, R. 2001, A\&A, 376, 982

Worley, C. E., \& Heintz, W. D. 1983, Publ. USNO 24, Pt. 7 